\title{
PASSIVE LEG RAISING EFFECT AT RESUSCITATION AMONG PATIENTS WITH VASOPLEGIC STATE
}

\author{
Panu Boontoterm*, Pusit Feungfoo**
}

"Division of Pulmonary and Critical Care Medicine, Department of Medicine, Phramongkutklao Hospital, Ratchawithi Road, Phayathai, Bangkok, Thailand 10400

**Department of Surgery, Phramongkutklao Hospital, Ratchawithi Road, Phayathai, Bangkok, Thailand 10400

\begin{abstract}
Background: Several dynamic parameters have been used clinically to predict volume responsiveness and to guide fluid administration of which passive leg raising (PLR) is one of the most reliable techniques. PLR induces rising in cardiac output attributes through an unstressed volume mobilization from legs to heart causing autologous preload increment. Appropriate fluid resuscitation is essential and can be optimized by hemodynamic-based approach to vasodilatory hypotension.

Objective: This study aimed to evaluate the effectiveness and safety of PLR at early resuscitation among patients with vasoplegia.

Methods: We conducted a comparison study concerning an experimental design using a single blinded assessment of the outcomes that assigned patients with shock to be treated with PLR or flat position at early resuscitation. Forty patients with shock were included in this study. Twenty patients performed PLR at early resuscitation compared with the others that performed in the flat position and were measured for cardiac output (CO), mean arterial pressure (MAP), diastolic blood pressure (DBP), heart rate (HR), central venous pressure (CVP) and systemic vascular resistance (SVR) immediately after the procedures. The primary outcome was to evaluate the effect of early PLR on hemodynamic variables among hypotensive patients by comparing the difference in $\mathrm{CO}$ while the secondary outcomes were differences in MAP, DBP, HR, CVP, SVR, survival at hospital admission and the pulmonary complications of chest $\mathrm{X}$-rays between the two groups.

Results: No difference was observed in baseline characteristics between the two groups of patients. Compared with the flat position, PLR at early resuscitation significantly increased CO (3.57 \pm 0.27 vs. $2.2 \pm 0.18 \mathrm{~L} / \mathrm{min}, p=0.037)$, MAP $(22.48 \pm 5.6$ vs. $10.83 \pm 4 \mathrm{mmHg}, p<0.001)$, DBP $(19 \pm 0.20$ vs. $1.23 \pm 0.12 \mathrm{mmHg}, p=0.001)$ and CVP $(4.52 \pm 0.19$ vs. $2.18 \pm 0.13 \mathrm{mmHg}, p=0.002)$. However, no differences were observed in HR, SVR, pulmonary complications of chest X-rays [2 (10\%) vs. 1 (5\%), $p=0.23]$ as well as survival at hospital admission [16 (80\%) vs. $13(65 \%), p=0.48]$ between the two groups. Conclusion: Among patients with shock, PLR at early resuscitation significantly increased CO, MAP, DBP and CVP than that of those performing the flat position. No differences were found in HR, SVR, pulmonary complications; PLR did not improve survival to hospital admission.
\end{abstract}

Keywords: Vasoplegia, Cardiac output, Venous return, Mean systemic pressure

J Southeast Asian Med Res 2021: 5(2):67-77

http://www.jseamed.org

Correspondence to:

Boontoterm P, Department of Surgery, Phramongkutklao Hospital, 315 Ratchawithi Road, Phayathai, Bangkok, Thailand 10400

Email:sapiens_panu@hotmail.com

Received: 11 September 2021

Revised: 14 October 2021

Accepted: 20 October 2021 


\section{INTRODUCTION}

Passive leg raising (PLR) is considered to be a maneuver that could promote venous return and increase systemic circulation. ${ }^{(1)}$ PLR at early resuscitation mimics rapid volume expansion and is often used in intensive care units during the hemodynamic assessment of patients. ${ }^{(2)}$ During vasoplegia or patients with shock, tissue perfusion is limited, leading to a low flow state. Increasing the venous and arterial bed resistances can improve myocardial and cerebral blood flow. ${ }^{(3)}$ Early PLR stresses the volume of the venous reservoir, increasing the mean systemic pressure (Pms), which is the driving pressure of the venous return flow. ${ }^{(4)}$ In a series of resuscitated pigs, PLR increased coronary perfusion pressure (CPP) compared with that in a control group. A retrograde volume loading of the aorta from early PLR may raise the intra-abdominal pressure and anterograde blood flow resistance, which increases the CPP gradient. ${ }^{(5)}$ PLR has been developed as a test to predict fluid responsiveness. ${ }^{(6)}$ This maneuver is supposed to transfer a significant volume of venous blood towards the intrathoracic compartment. However, it has been suggested that PLR could have nonsignificant effects on cardiac preload, in particular in the case of intra-abdominal hypertension. This would result in a negative PLR test result in spite of actual fluid responsiveness.

In recent years, concern has been growing about the safety of various interventions performed at early resuscitation. Fluid infusion at early resuscitation has led to worsened clinical outcomes. ${ }^{(7,8)}$ Another resuscitation body position, such as Trendelenburg, was associated with increased intracranial pressure. Data about the safety of PLR and the beneficial effect of early PLR remain limited. We hypothesized that early PLR could be a safe maneuver and could improve survival at discharge compared with that of patients treated using a standard protocol.

\section{Methods}

This study was reviewed and approved by the Ethics Committee of Institutional Review Board, Royal Thai Army Medical Department (IRBRTA 292/2563). The study was conducted in accordance with the Council for International Organization of Medical Science (CIOMS) Guidelines 2012 and Good Clinical Practices.

Sample size was calculated according to the comparison studies of PLR and the flat position in volume expansion. ${ }^{(4,15)}$ At least 26 patients in total were required to compare differences between the two groups. Patients were enrolled on the scene at the initial shock and resuscitation assessment. In this study, patients with shock were included. A single blinded experiment was conducted by randomization of $1: 1$ ratio using variables of block size, computer-generated sequence and allocation and kept in opaque envelopes.

\section{Participants}

Informed consent was obtained from the enrolled patients or their legal representatives. From May 2020 to May 2021, 40 patients admitted in Medical Intensive Care Unit (ICU), Phramongkutklao Hospital, were monitored for invasive arterial blood pressure, peripheral $\mathrm{O}_{2}$ saturation $\left(\mathrm{SpO}_{2}\right)$, and electrocardiogram.

Patients were assigned to PLR and the flat position groups. Of these, 20 patients used PLR which was performed within the first $5 \mathrm{~min}$ after initial shock and resuscitation assessment and was maintained until the end of resuscitation or until the patients presented stable hemodynamics. The angle of PLR was set at $45^{\circ}$ following a related report. ${ }^{(4)}$ To ensure that the legs were lifted at this angle, different assays were made. As a result, all ICU beds were equipped and adjusted to accurately measure this angle.

All patients were measured for $\mathrm{CO}$, mean arterial pressure (MAP), diastolic blood pressure (DBP), heart rate (HR), central venous pressure (CVP) and systemic vascular resistance (SVR) immediately after PLR procedures. The inclusion criteria were patients aged more than 18 years old. The exclusion criteria included patients who had contraindications for PLR, e.g., limb amputation, traumatic patients with suspected pelvic or lower limb fracture, pregnancy, intraabdominal pressure more than $16 \mathrm{mmHg}$, increased intracranial pressure and pneumothorax. The primary outcome was to evaluate the effect of early PLR on hemodynamic variables among hypotensive 
patients by comparing the difference of $\mathrm{CO}$ while the secondary outcomes were to compare differences in MAP, DBP, HR, CVP and SVR, survival at hospital admission and pulmonary complications in chest $\mathrm{x}$-rays between the two groups.

Shock was defined as persistent MAP less than $65 \mathrm{mmHg}$ at least $15 \mathrm{~min}$ despite adequate volume resuscitation (performed dynamic parameters shows fluid nonresponsive) or required vasopressors to maintain MAP more than 65 $\mathrm{mmHg}$.

Septic shock was defined according to the Third International Consensus Definitions for Sepsis and Septic Shock (Sepsis-3) (persistence of infection, and required vasopressors to optimize $\mathrm{MAP} \geq 65 \mathrm{mmHg}$, combined with a serum lactate level $>2 \mathrm{mmoL} / \mathrm{L}(18 \mathrm{mg} / \mathrm{dL})$ despite adequate volume resuscitation.

Resuscitation was performed using early intravenous fluid resuscitation with balanced salt solution crystalloid or isotonic crystalloid at least $30 \mathrm{~mL} / \mathrm{kg}$ according to Surviving Sepsis Campaign guidelines 2018, European Society of Intensive Care Medicine guidelines. Dynamic parameters used to assess adequate fluid resuscitation included pulse pressure variation (PPV), stroke volume variation (SVV) or mini-fluid challenge test when performed dynamic parameters showed fluid nonresponsive, and early use of vasopressor (norepinephrine) to maintain MAP more than 65 mmHg was applied.

\section{General management in ICU}

Patients were sedated with fentanyl and mechanically ventilated using pressure-controlled ventilation, aimed to maintain at Pplat $<30 \mathrm{~cm}$ $\mathrm{H}_{2} \mathrm{O}$, using a tidal volume $\left(\mathrm{V}_{\mathrm{T}}\right)$ of $6-8 \mathrm{~mL} / \mathrm{kg}$ of predicted body weight. The faction of inspired oxygen $\left(\mathrm{FiO}_{2}\right)$ and Positive-End Respiratory Pressure (PEEP) were titrated to achieve peripheral saturation of oxygen $\left(\mathrm{SpO}_{2}\right)$ more than $94 \%$, and RR was set to maintain arterial partial pressure of carbon dioxide $\left(\mathrm{PaCO}_{2}\right)$, and 35-45
$\mathrm{mmHg}$ end-tidal carbon dioxide $\left(\mathrm{ETCO}_{2}\right)$ was continuously measured.

\section{Hemodynamic monitoring}

Radial arterial catheter and central venous catheter were linked to a bedside monitor on one side and to a specific transducer (Philips Intellivue Philips MX600, USA) for blood pressure, DBP, HR and CVP monitoring. The values of $\mathrm{CO}$ and SVR were estimated from pulse contour analysis (EV1000 clinical platform, Edwards advanced hemodynamic monitoring tools for an integrated Edwards Critical Care System, USA).

\section{Interventions}

PLR was performed within the first $5 \mathrm{~min}$ after acute circulatory failure at the ICU and was maintained until the end of resuscitation. Procedures involved patients sitting in the 45 degrees, head up, semi-recumbent position, then lowering the patient's upper body to horizontal and passively raising the legs at 45 degrees up then maintaining the maximal effect occurring during resuscitation. To assess postresuscitation pulmonary complications, the report of the attending physician or radiologist on the first X-ray taken at the ICU was evaluated. Lung complications were considered when bilateral lung opacities, edema, pulmonary congestion or bilateral alveolar pattern and survival at hospital admission were described.

\section{Statistical analysis}

Results were expressed as mean \pm SD when data were normally distributed or median and interquartile range (IQR) if not. Hemodynamic parameters were compared between PLR and the flat position during resuscitation using the independent-t test, paired t-test, Fisher's exact test, Pearson's correlation, and repeated measure ANOVA test. The effects of volume expansion on hemodynamic parameters were analyzed using the Friedman nonparametric repeated measures comparisons. A $p$-value less than 0.05 was considered to be statistically significant. Statistical analysis was performed using IBM SPSS Statistics for Windows, Version 23.0. (Armonk, NY, IBM Corp.) 


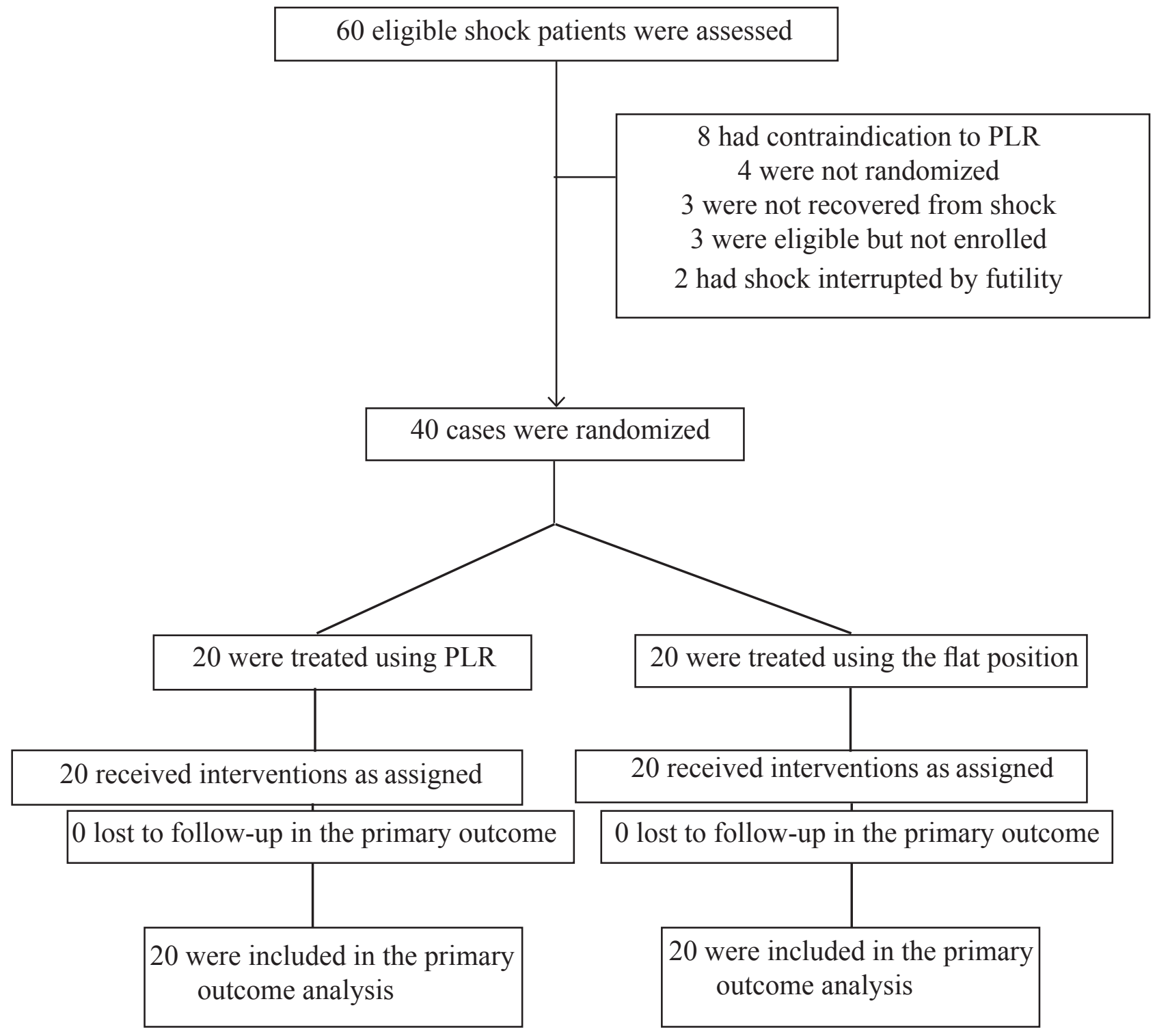

Figure 1. Flow chart of patient enrollment and analysis in the trial

\section{Results}

\section{Patient characteristics}

During the study period, 40 patients with acute circulatory failure were included. Twenty patients performed PLR. All patients were measured for CO, MAP, DBP, HR, CVP and SVR immediately after the procedures. Most patients were female $(52 \%)$ with average age of 68 years. The most frequent coexisting disease was hypertension while the most frequent etiology of shock was septic shock (Table 1).
Regarding adverse effects, the incidence of pulmonary complications of the first chest X-rays were similar between the PLR and flat position groups, $(10 \%$ vs. $5 \%, p=0.23)$.

\section{Clinical outcomes}

No significant differences in survival at hospital admission were found [16 (80\%) vs. 13 $(65 \%), p=0.48]$ between the two groups. 
Table 1. Demographic data of 40 patients with acute circulatory failure

\begin{tabular}{ll}
\hline Demographic data & $\mathrm{N}=40$ \\
\hline Male, $\mathrm{n}(\%)$ & $19(47.5)$ \\
Female, $\mathrm{n}(\%)$ & $21(52.5)$ \\
Age $(\mathrm{yr})$ & $68.25 \pm 17.23$ \\
Body weight $(\mathrm{kg})$ & $58.25 \pm 5.38$ \\
Co-morbidity, $\mathbf{n}(\mathbf{\%})$ & \\
Hypertension & $29(72.5)$ \\
Dyslipidemia & $18(45)$ \\
Diabetes mellitus & $18(45)$ \\
Chronic kidney disease & $10(25)$ \\
Chronic liver disease & $9(22.5)$ \\
Coronary artery disease & $4(10)$ \\
Other diseases & $21(52.5)$ \\
IV fluid (mL) & $1725 \pm 521$ \\
Type of shock, $\mathbf{n}(\%)$ & \\
Septic & $33(82.5)$ \\
Cardiogenic & $4(10)$ \\
Hypovolemic & $3(7.5)$ \\
\hline
\end{tabular}

Characteristics between the two groups of patients

No significant difference of baseline characteristics was observed between the two groups of patients (Table 2).

Table 2. Comparison baseline characteristics between the two patient groups

\begin{tabular}{lccc}
\hline \multicolumn{1}{c}{ Characteristics } & $\begin{array}{c}\text { PLR } \\
(\mathbf{n = 2 0 )}\end{array}$ & Flat position (n=20) & p-value \\
\hline Male, n (\%) & $9(45)$ & $10(50)$ & 1.0 \\
Female, n (\%) & $11(55 \%)$ & $10(50 \%)$ & 1.0 \\
Age (yr) & $66.1 \pm 18.5$ & $70.4 \pm 16.04$ & 0.437 \\
Body weight (kg) & $58.5 \pm 5.4$ & $58 \pm 5.48$ & 0.773 \\
Coexisting diseases, n (\%) & $16(80)$ & & \\
Hypertension & $10(50)$ & $13(65)$ & 0.480 \\
Dyslipidemia & $10(50)$ & $8(40)$ & 0.751 \\
Diabetes mellitus & $8(40)$ & $8(40)$ & 0.751 \\
Chronic kidney disease & $4(20)$ & $2(10)$ & 0.065 \\
Chronic liver disease & $4(20)$ & $0(25)$ & 1.0 \\
Coronary artery disease & $12.7 \pm 1.95$ & $12.65 \pm 2.23$ & 0.106 \\
APACHE II Score & $1651.5 \pm 470.15$ & $1798 \pm 570.53$ & 0.940 \\
Received IV fluid (mL) & $6(30 \%)$ & $8(40 \%)$ & 0.381 \\
Fever & & & 0.741 \\
Sedation drug & & & \\
\hline
\end{tabular}


Table 2. Comparison baseline characteristics between the two patient groups (ext.)

\begin{tabular}{lccc}
\hline \multicolumn{1}{c}{ Characteristics } & $\begin{array}{c}\text { PLR } \\
(\mathbf{n = 2 0})\end{array}$ & Flat position (n=20) & p-value \\
\hline Fentanyl & $17(85 \%)$ & $16(80 \%)$ & 1 \\
Heart rate (beat/min ) & $94 \pm 23.25$ & $93 \pm 16.76$ & 0.849 \\
MAP on admission $(\mathrm{mmHg})$ & $54.95 \pm 4.68$ & $57.3 \pm 6.5$ & 0.67 \\
DBP on admission $(\mathrm{mmHg})$ & $52 \pm 5.55$ & $54 \pm 4.76$ & 0.139 \\
CVP on admission $(\mathrm{mmHg})$ & $7.68 \pm 1.49$ & $8.1 \pm 1.8$ & 0.73 \\
CO on admission $(\mathrm{L} / \mathrm{min})$ & $4.4 \pm 1.27$ & $4.8 \pm 1.1$ & 0.81 \\
SVR on admission $\left(\right.$ dyn.s/cm $\left.{ }^{5}\right)$ & $870 \pm 43.23$ & $877 \pm 45.41$ & 0.576 \\
Blood lactate on admission $(\mathrm{mmol} / \mathrm{L})$ & $5.0(4.0-6.9)$ & $4.5(3.0-7.5)$ & 0.79 \\
Type of Shock & & & \\
$\quad$ Septic & $17(85)$ & $16(80)$ & 1 \\
$\quad$ Cardiogenic & $2(10)$ & $2(10)$ & 0.605 \\
$\quad$ Hypovolemic & $2(10)$ & $1(5)$ & 0.231 \\
Dose NE $(\mu \mathrm{g} / \mathrm{kg} / \mathrm{min})$ & $0.34 \pm 0.07$ & $0.35 \pm 0.06$ & 0.486 \\
Survival at hospital admission & $16(80)$ & $13(65)$ & 0.480 \\
Pulmonary complications & $2(10)$ & $1(5)$ & 0.231 \\
Days in ICU (mean) & 5 & 6 & 0.63 \\
\hline
\end{tabular}

Values presented as mean \pm SD or $n(\%), p$-values corresponded to independent-t test and Fisher's exact test.

*Acute Physiology and Chronic Health Evaluation

Differences of CO, MAP, DBP, HR, CVP and SVR compared between PLR and flat position groups

Changes in hemodynamic variables are shown in Table 3. PLR significantly increased $\mathrm{CO}(3.57 \pm 0.27$ vs. $2.2 \pm 0.18 \mathrm{~L} / \mathrm{min}, p=0.037)$, $\operatorname{MAP}(22.48 \pm 5.6$ vs. $10.83 \pm 4 \mathrm{mmHg}, p<0.001)$, $\mathrm{DBP}(19 \pm 0.20$ vs. $1.23 \pm 0.12 \mathrm{mmHg}, p=0.001)$ and CVP (4.52 \pm 0.19 vs. $2.18 \pm 0.13 \mathrm{mmHg}$, $p=0.002)$. PLR increased CO, MAP and CVP during resuscitation from baseline. Compared with the flat position, PLR increased CO, MAP and CVP more. No significant differences were found between the two groups regarding SVR [6 $(-27.34,15.34)$ vs. $1(-46.83,48.83)$ dyn.s/ $/ \mathrm{cm}^{5}$ $(95 \% \mathrm{CI}), p=0.704]$ and HR $[0.67 \pm 0.15$ vs. 0.2 \pm 0.35 beat $/ \mathrm{min}, p=0.98]$.

Table 3. Changes in hemodynamic variables from baseline in PLR and the flat position during resuscitation.

\begin{tabular}{lccccc}
\hline \multicolumn{1}{c}{ Variables } & $\begin{array}{c}\text { Baseline before } \\
\text { PLR } \\
(\mathrm{n}=20)\end{array}$ & $\begin{array}{c}\text { PLR } \\
(\mathrm{n}=20)\end{array}$ & $\begin{array}{c}\text { Baseline before } \\
\text { flat position } \\
(\mathrm{n}=20)\end{array}$ & $\begin{array}{c}\text { Flat position } \\
(\mathrm{n}=20)\end{array}$ & $\boldsymbol{p}$-value \\
\hline CO & $4.4 \pm 1.27$ & $7.97 \pm 1.54$ & $4.8 \pm 1.1$ & $7.0 \pm 1.28$ & $0.037^{\#}$ \\
$(\mathrm{~L} / \mathrm{min})$ & $\begin{array}{c}\text { Mean change from } \\
\text { baseline }\end{array}$ & $3.57 \pm 0.27$ & $\begin{array}{c}\text { Mean change from } \\
\text { baseline }\end{array}$ & $2.2 \pm 0.18$ & \\
& & $\mathrm{p}<0.001^{*}$ & & $p<0.001^{*}$ & \\
\hline MAP & $54.95 \pm 4.68$ & $77.43 \pm 7.78$ & $57.3 \pm 6.5$ & $68.13 \pm 2.5$ & $<0.001^{\#}$ \\
$(\mathrm{mmHg})$ & Mean change & $22.48 \pm 5.6$ & $\begin{array}{c}\text { Mean change from } \\
\text { baseline }\end{array}$ & $10.83 \pm 4$ & \\
& baseline & $p<0.001^{*}$ & & $p<0.001^{*}$ & \\
\hline
\end{tabular}


Table 3. Changes in hemodynamic variables from baseline in PLR and the flat position during resuscitation (ext.)

\begin{tabular}{|c|c|c|c|c|c|}
\hline Variables & $\begin{array}{c}\text { Baseline before } \\
\text { PLR } \\
(\mathrm{n}=20)\end{array}$ & $\begin{array}{c}\text { PLR } \\
(\mathrm{n}=20)\end{array}$ & $\begin{array}{c}\text { Baseline before } \\
\text { flat position } \\
(\mathrm{n}=20)\end{array}$ & $\begin{array}{l}\text { Flat position } \\
\qquad(\mathrm{n}=20)\end{array}$ & $p$-value \\
\hline $\begin{array}{l}\text { DBP } \\
(\mathrm{mmHg})\end{array}$ & $\begin{array}{c}52 \pm 5.35 \\
\text { Mean change from } \\
\text { baseline }\end{array}$ & $\begin{array}{l}71 \pm 5.55 \\
19 \pm 0.20 \\
p<0.001^{*}\end{array}$ & $\begin{array}{c}54 \pm 4.76 \\
\text { Mean change from } \\
\text { baseline }\end{array}$ & $\begin{array}{r}55.77 \pm 4.88 \\
1.23 \pm 0.12 \\
p=0.139\end{array}$ & $0.001^{\#}$ \\
\hline $\begin{array}{l}\text { HR } \\
\text { (beat } / \text { min ) }\end{array}$ & $\begin{array}{c}94 \pm 23.25 \\
\text { Mean change from } \\
\text { baseline }\end{array}$ & $\begin{array}{c}93.33 \pm 23.4 \\
0.67 \pm 0.15 \\
p=0.849\end{array}$ & $\begin{array}{c}93 \pm 16.76 \\
\text { Mean change from } \\
\text { baseline }\end{array}$ & $\begin{array}{c}93.2 \pm 16.41 \\
0.2 \pm 0.35 \\
p=0.92\end{array}$ & 0.98 \\
\hline $\begin{array}{l}\text { CVP } \\
(\mathrm{mmHg})\end{array}$ & $\begin{array}{c}7.68 \pm 1.49 \\
\text { Mean change from } \\
\text { baseline }\end{array}$ & $\begin{array}{l}12.2 \pm 1.68 \\
4.52 \pm 0.19 \\
p<0.001^{*}\end{array}$ & $\begin{array}{c}8.1 \pm 1.8 \\
\text { Mean change from } \\
\text { baseline }\end{array}$ & $\begin{array}{c}10.28 \pm 1.93 \\
2.18 \pm 0.13 \\
p<0.001^{*}\end{array}$ & $0.002^{\#}$ \\
\hline $\begin{array}{l}\text { SVR } \\
(\text { dyn.s/cm })\end{array}$ & $\begin{array}{c}870 \pm 43.23 \\
\text { Mean change from } \\
\text { baseline } \\
(95 \% \mathrm{CI})\end{array}$ & $\begin{array}{c}876 \pm 39.26 \\
6 \\
(-27.34,15.34) \\
p=0.576\end{array}$ & $\begin{array}{c}877 \pm 45.41 \\
\text { Mean change from } \\
\text { baseline } \\
(95 \% \mathrm{CI})\end{array}$ & $\begin{array}{c}878 \pm 45.05 \\
1 \\
(46.83,48.83) \\
p=0.98\end{array}$ & 0.53 \\
\hline
\end{tabular}

Values presented as mean $\pm \mathrm{SD}$ and mean change presented as mean $\pm \mathrm{SD}$ and interquartile range, *depicts $p<0.05$ and compared between baseline vs. each intervention

\# depicts $p<0.05$ and compared between two interventions

$P$-values were analyzed using the paired t-test and independent t-test.

\section{Discussion}

In this study, baseline characteristics of each group were comparable. We evaluated the effects of PLR on CO, MAP, DBP, HR, CVP and SVR among patients with acute circulatory failure during resuscitation. We found that PLR increased $\mathrm{CO}$, MAP, DBP and CVP during resuscitation from baseline. Additionally, when compared with the flat position, PLR increased CO, MAP, DBP and CVP more. Significantly increased CO, MAP, DBP and CVP confirmed that PLR could actually represent a powerful preload challenge. The increase of venous return was attested to the increase of CO. Interestingly, PLR did not reduce venous return resistance (Rvr) while a decrease in Rvr due to a reduced sympathetic tone could have been expected from an improvement in CO. PLR resulted in a larger increase in Pms than in CVP. This increase in the pressure gradient for venous return was associated with an increase in
CO. PLR effected increased venous return; thus, $\mathrm{CO}, \mathrm{MAP}$ and CVP values increased when $\mathrm{CO}$ and MAP increased. This could improve tissue perfusion, tissue oxygenation and promote recovery of shock.

A Swedish research group reported that PLR was performed more often in cases involving a worsened clinical scenario and early PLR could improve its benefit on survival. ${ }^{(9)}$ The idea of a transient effect of PLR over time has been described among patients with septic shock and is attributed to capillary leak. ${ }^{(10)}$ During acute circulatory failure, maintained tissue perfusion which could favor the shortened effect of PLR on $\mathrm{CO}^{(11)}$ Optimizing venous return is the key to improve survival outcomes.

Experimental data support the distinct hemodynamic effect of PLR and volume load during resuscitation. Volume loading has been associated with decreased CPP due to the detrimental effect 
of the increase in right atrial pressure (RAP) during the decompression phase. ${ }^{(12)}$ However, PLR seemed not to alter RAP and has been associated with an increase in CPP. ${ }^{(13)}$ It should be considered that the greatest change in $\mathrm{CO}$ due to PLR occurred after $1 \mathrm{~min}$ of the procedure. ${ }^{(14)}$

In the present study, we aimed to assess how hemodynamic variables changed during PLR among patients with shock. In particular, we aimed to investigate whether the absence of increased CO during PLR was due to an absence of increase in venous return, resulting in the absence of a significant increase in cardiac preload, or to a preload independence per se, that is, to an absence of increased $\mathrm{CO}$ to a significant increase in cardiac preload.

Laurent Guerin et al. ${ }^{(15)}$ conducted a passive leg raising study among patients with shock and hemodynamic effects of PLR showing thatPLR increased cardiac index (CI) by $17 \pm 20 \%$. During PLR, CVP and CI significantly increased. PLR did not change the intra-abdominal pressure among the whole subjects $(14 \pm 6 \mathrm{mmHg}$ before vs. $13 \pm 5 \mathrm{mmHg}$ during PLR, $p=0.26$ ) or among patients with intra-abdominal hypertension at baseline $(17 \pm 4 \mathrm{mmHg}$ before vs. $16 \pm$ $4 \mathrm{mmHg}$ during PLR, $p=0.14$ ). Considering the whole subjects, the PLR-induced changes in CI predicted fluid responsiveness with an area under the receiver operator characteristic (ROC) curve of $0.98 \pm 0.03$. Our results confirmed the results to a related study showing $54 \%$ of increased CO. PLR could be used as a test for predicting fluid responsiveness. The test assumes that it increases the stressed blood volume by inducing the gravitational transfer of venous blood from the inferior limbs and the splanchnic compartment toward the cardiac cavities. ${ }^{(18)}$ Nevertheless, the effects of PLR on the determinants of venous return have been investigated in only one study. (19) PLR test significantly shifts intravascular fluid from the legs to the abdomen, suggesting that this dynamic test may not be appropriate among patients with risk of intra-abdomenal or intrathoracic hypertension and also patients at risk of high intracranial pressure. Moreover, other studies have suggested that the PLR test would not be reliable in the case of intraabdominal hypertension because it would compress the inferior vena cava. ${ }^{(20,21)}$ However, in this study, we excluded patients with intra-abdominal hypertension.

In these regards, our study provides some interesting issues on the hemodynamic effects of PLR that PLR induced significantly increases in $\mathrm{CO}$ and CVP among all patients, confirming that it could actually represent a powerful preload challenge. These results agree with those of Keller et al. ${ }^{(19)}$, who reported that PLR increased CVP from 4 to $6 \mathrm{mmHg}$. One of major interests of the study was to analyze the effects of PLR depending on the fluid responsiveness status. Increasing the pressure gradient for venous return was associated with an increase in CO. Physiologically, Pms depends on vascular compliance and on the volume of venous blood that is submitted to the strain of the venous reservoir walls, i.e., stressed blood volume. ${ }^{(22)}$ As fluid infusion is assumed not to alter vascular compliance, our results suggested that fluid infusion increased Pms and cardiac preload by increasing the stressed blood volume, confirming the results by Keller et al. ${ }^{(19)}$ Our results suggest that PLR also increased the stressed blood volume. CVP did not increase as much as Pms during PLR. This was probably related to the fact that in these fluid responsive patients, the heart was working on the steep part of the Frank-Starling curve. Therefore, a rightward shift on the venous return curve induced by the increase in Pms resulted in a smaller increase in CVP. Interestingly, PLR did not reduce venous return resistance (Rvr), while a decrease in Rvr due to a reduction in the sympathetic tone could have been expected from an improvement in CO.

Another physiological advantage was significantly higher diastolic blood pressure induced by PLR; the major determinant of coronary blood flow. One potential benefit is the combined increased diastolic blood pressure and steady heart rate theoretically allows more balance in myocardial oxygen demand and supply among patients with septic shock. The rapid effect of PLR as internal volume resuscitation has gained more attentions in its effectiveness and safety for out-of-hospital cardiopulmonary resuscitation (CPR) setting, however current evidence has not revealed clinical benefits. 
A related study on PLR revealed significant sustained effects on cardiovascular parameters even at 10 min after the start of PLR. ${ }^{(23)}$ Out of the parameters observed in this study, $\mathrm{CO}$ showed the largest and most stable sustained increase during the entire PLR time course. CO promptly returned to pre-PLR values at the end of PLR. This increase was believed to be primarily a passive response to the increase in preload due to PLR, which increases CVP, resulting in increased right ventricular $\mathrm{CO}$ and subsequently that of the left ventricular preload. This increase in CO presumably caused increases in the other parameters, including MAP, SBP and DBP during PLR. Wong et al. ${ }^{(24)}$ reported that patients whose 500 $\mathrm{mL}$ blood was extracted before PLR showed a significantly greater increase in $\mathrm{CO}$ than that of the control. These results, together with our present observation, suggested that PLR could be an effective procedure for patients with relatively normal cardiac functions who are in hypovolemic shock and the vasoplegic state. HR tended to decrease in response to PLR. Among normal patients, strong homeostatic mechanisms work to maintain constant blood flow to vital organs. The increase in CO caused by PLR may have induced a blood pressure increase that was sensed by carotid or cardiopulmonary baroreceptors; and thus, decreased HR through a negative feedback system ${ }^{(25-27)}$. The decrease in HR during PLR was interpreted as the result of a compensatory reflex evoked by the increased $\mathrm{CO}$ in response to PLR. SVR is another important parameter of cardiovascular function. Although SVR gradually increased after a transient decrease at the start of PLR, no significant changes in SVR were observed at any time. This SVR response to PLR appeared to be transient and compensatory to the rapid increase in $\mathrm{CO}$, which decayed in a relatively short time and caused no significant differences at any time.

Our study revealed the potential role of PLR in ICU that is probably not only the test of volume responsiveness but also an intervention for volume resuscitation among patients in the vasoplegic state even though the hemodynamic effect may last only a short period.
Study limitations are discussed below. Firstly, CO was monitored using the EV1000 clinical platform, performed by analyzing the radial artery pressure waveform. For rapid changing of intravascular volume or when using vasopressors, monitoring of femoral artery pressure has been recommended. Secondly, our study was conducted in a single center and confined only to patients with shock, so our findings could not be applied for other critically-ill patients without need of circulatory supports. Third, the time between shock with initial resuscitation and the PLR was not recorded which could be a potential confounder. Finally, PLR could have stimulated sympathetic tone and interfered with hemodynamic interpretation.

In this study, PLR was considered to be a maneuver that could promote venous return and improve hemodynamic variable among patients with shock. Some situations, i.e., CPR during cardiac arrest, can increase venous return and artificial circulation during chest compressions. During CPR, CO is limited; increasing the venous and arterial bed resistance that can improve myocardial and cerebral blood flow. PLR stresses the volume of the venous reservoir, increasing the mean systemic filling pressure, which is the driving pressure of the venous return flow. Use of coronary perfusion pressure (CPP) is a good predictor of the return of spontaneous circulation (ROSC) because a retrograde volume loading of the aorta from the PLR may occur, raising the intra-abdominal pressure and the anterograde blood flow resistance, which increases the CPP gradient. Further study about safety of various interventions performed by emergency teams during resuscitation would be very helpful. Data about the safety of PLR during CPR, and the beneficial effect of PLR performed during CPR remains very limited. It can be hypothesized that PLR performed at the beginning of out-ofhospital cardiac arrest treatment would be a safe maneuver and improve survival at discharge with good neurological outcomes compared with those of patients treated using a standard protocol.

\section{Conclusion}

Among patients with acute circulatory failure, PLR at early resuscitation significantly increased 
CO, MAP, DBP and CVP more than those using the flat position. No differences in HR, SVR and pulmonary complications have been found; PLR did not improve survival to hospital admission.

\section{Availability of data and materials}

The datasets used and/or analyzed during the current study are available from the corresponding author on reasonable request.

\section{Conflict of interests}

No potential conflict of interest relevant to this article was reported.

\section{Acknowledgements}

We would like to thank Mrs Dollapas Panpanich for her assistance on data analysis, and also extend our appreciation to the staff of the Surgical and Medical Intensive Care Unit, Phramongkutklao Hospital for their assistance and resources used in conducting the study.

\section{REFERENCES}

1. Standards and guidelines for Cardiopulmonary Resuscitation (CPR) and Emergency Cardiac Care (ECC). JAMA 1986; 255: 2905-84.

2. RutlenDL,WackersFJ,ZaretBL. Radionuclide assessment of peripheral intravascular capacity: a technique to measure intravascular volume changes in the capacitance circulation in man. Circulation 1981; 64: 146-52.

3. Yang Z, Tang D, Wu X, Hu X, Xu J, Qian J, et al. A tourniquet assisted cardiopulmonary resuscitation augments myocardial perfusion in a porcine model of cardiac arrest. Resuscitation 2015; 86: 49-53.

4. Monnet X, Teboul J-L. Passive leg raising. Intensive Care Med 2008; 34: 659-63.

5. Dragoumanos V, Iacovidou N, Chalkias A, Lelovas P,Koutsovasilis A, Papalois A, et al. Passive leg raising during cardiopulmonary resuscitation results in improved neurological outcome in a swine model of prolonged ventricular fibrillation. Am J Emerg Med 2012; 30: 1935-42.

6. Keller G, Desebbe O, Benard M, Bouchet JB, Lehot JJ. Bedside assessment of PLR effects on venous return. J Clin Monit Comput 2011; 25: 257-63.
7. Bernard SA, Smith K, Finn J, Hein C, Grantham H, Bray JE, et al. Induction of therapeutic hypothermia during out-of-hospital cardiac arrest using a rapid infusion of cold saline: the RINSE trial (Rapid Infusion of Cold Normal Saline). Circulation 2016; 134: 797-805.

8. Kim F, Nichol G, Maynard C, Hallstrom A, Kudenchuk PJ, Rea $T$, et al. Effect of prehospital induction of mild hypothermia on survival and neurological status among adults with cardiac arrest: a randomized clinical trial. JAMA 2014; 311: 45.

9. Holmén J, Herlitz J, Jimenez-Herrera M, Karlsson T, Axelsson C. Passive leg raising in out-of-hospital cardiac arrest. Resuscitation 2019; 137: 94-101.

10. Monnet X, Rienzo M, Osman D, Anguel N, Richard C, Pinsky MR, et al. Passive leg raising predicts fluid responsiveness in the critically ill. Crit Care Med 2006; 34: 1402-7.

11. Chalkias A, Xanthos T. Pathophysiology and pathogenesis of post resuscitation myocardial stunning. Heart Fail Rev 2011; 17: 117-28.

12. Ditchey RV. Potential adverse effects of volume loading on perfusion of vital organs during closed-chest resuscitation.Circulation 1984; 69: 181-9.

13. Paradis NA. Coronary perfusion pressure and the return of spontaneous circulation in human cardiopulmonary resuscitation. JAMA 1990; 263: 1106.

14. Axelsson C, Holmberg S, Karlsson T, Axelssonå $\mathrm{B}$, Herlitz J. Passive leg raising during cardiopulmonary resuscitation in out-of-hospital cardiac arrest-Does it improve circulation and outcome? Resuscitation 2010; 81: 1615-20.

15. Guérin L, Teboul JL, Persichini R, Dres M, Richard C, Monnet X. Effects of passive leg raising and volume expansion on mean systemic pressure and venous return in shock in humans. Crit Care 2015; 19: 411.

16. Mitchell M. levy, Laura E. Evans, Andrew Rhodes. The surviving sepsis campaign bundle: 2018 update. Intensive Care Med 2018; 44: 925-28. 
17. Laurent Guerin, Jean-Louis, Romain Persichini, Effect of passive leg raising and volume expansion on mean systemic pressure and venous return. J Anesthesiol 2018; 71: 386-93.

18. Monnet X, Teboul JL. Passive leg raising: five rules, not a drop of fluid. Crit Care. 2015; 19: 18.

19. Keller G, Desebbe O, Benard M, Bouchet JB, Lehot JJ. Bedside assessment of passive leg raising effects on venous return. J Clin Monit Comput 2011; 25: 257-63.

20. Malbrain ML, Reuter DA. Assessing fluid responsiveness with the passive leg raising maneuver in patients with increased intraabdominal pressure: be aware that not all blood returns Crit Care Med 2010; 38: 1912-5.

21. Mahjoub Y, Touzeau J, Airapetian N, Lorne E, Hijazi M, Zogheib E, et al. The passive leg-raising maneuver cannot accurately predict fluid responsiveness in patients with intra-abdominal hypertension. Crit Care Med 2010; 38: 1824-9.

22. Teboul JL. Mean systemic pressure: we can now estimate it, but for what. Intensive Care Med 2013; 39: 1487-8.
23. Yoshioka A, Nishimura K, Seki K, Onodera $\mathrm{N}$ Effects of passive leg raising on cross sectional area of inferior vena cava and stroke volume. Kawasaki Med Welfare J 2010; 19: 285-90.

24. Wong DH, O'Connor D, Tremper KK, Zaccari J, Thompson P, et al. Changes in cardiac output after acute blood loss and position change in man. Crit Care Med 1989; 17: 979-83.

25. RoddieIC, Shepherd JT. The effects of carotid artery compression in man with special reference to changes in vascular resistance in the limbs. J Physiol 1957; 139: 377-84.

26. Barbieri R, Triedman JK, Saul JP. Heart rate control and mechanical cardiopulmonary coupling to assess central volume: a systems analysis. Am J Physiol Regul Integr Comp Physiol 2002; 283: R1210-20.

27. Parati G, Grassi G, Coruzzi P, Musiari L, Ravogli A, et al. Influence of cardiopulmonary receptors on the bradycardic responses to carotid baroreceptor stimulation in man. Clin Sci (Lond) 1987; 72: 639-45. 